\title{
Effects of early lactation concentrate level and glucogenic feed on feed intake, milk production and energy metabolism in dairy cows and heifers*
}

\author{
T. Kokkonen', A. Tesfa ${ }^{1}$, M. Tuori' ${ }^{1}$ K. Hissa ${ }^{2}$, E. Jukola ${ }^{2}$ and \\ L. Syrjälä-Qvist ${ }^{1}$
}

'Department of Animal Science, University of Helsinki

P.O.B 28,00014 Helsinki. Finland

${ }^{2}$ Suomen Rehu Lud.

P.O.B 105, 0024/ Ilelsinki, Finland

(Received 10 May 2000: accepted 4 October 2000)

\section{ABSTRACT}

In order to examine the effect of two levels of concentrate and the effect of glucogenic feed $(0 \mathrm{l} / \mathrm{d}(\mathrm{G} 0)$ or $1 \mathrm{l} / \mathrm{d}(\mathrm{G} 1)) 16$ multiparous and 16 primiparous Friesian cows were used in a factorial $2 \times 2$ design. The concentrate levels for multiparous cows were $11 \mathrm{~kg} / \mathrm{d}(\mathrm{Cll})$ and $15 \mathrm{~kg} / \mathrm{d}(\mathrm{C} 15)$ and for primiparous cows $9 \mathrm{~kg} / \mathrm{d}(\mathrm{C} 9)$ and $12 \mathrm{~kg} / \mathrm{d}(\mathrm{Cl2})$. The glucogenic feed consisted of propylenc glycol, polyols, molasses and niacin. All the animals were offered wilted silage ad lihitum. The experimental period started from calving and lasted for 12 wecks.

Multiparous cows substituted concentrate for silage when glucogenic feed was not given whereas with glucogenic feed the effect of concentrate on silage dry matter intake (DMI) was minor. Glucogenic feed had a minor effect on milk production at the higher level of concentrate and a negative effect on milk production at the lower level of concentrate. Due to this interaction the milk yield response to the higher level of concentrate was higher with glucogenic feed. The low rumen degradable protein supply in $\mathrm{CllGl}$ was probably the cause of these interactions.

The milk production response of primiparous cows to the higher level of concentrate was low. After peak yield the plasma insulin concentrations were higher with the higher concentrate level. This was accompanied by higher liveweight gain.

In conclusion. the response of multiparous cows to higher concentrate level depended on the supply of rumen degradable protein, whereas the comparable response of priniparous cows was

\footnotetext{
* Financial support by the $\Lambda$ gricultural Research Foundation of August Johannes and Aino Tiura
} 
limited by milk yield potential and the needs of growth. The glucogenic feed showed an ability to increase plasma glucose and decrease the concentrations of ketones.

KEY WORDS: dairy cows, early lactation, grass silage, energy intake, energy metabolism

\section{INTRODUCTION}

The feed intake capacity of early lactation cows increases more slowly than their milk yield. Therefore these cows are in negative energy balance for several weeks after parturition. This energy deficit can be partly alleviated by increasing the amount and the proportion of concentrates in feeding. Increased concentrate intake has led to higher milk yield, higher concentration of milk protein and lower concentration of milk fat (Thomas et al., 1986; Aston et al., 1995) with diminishing returns at higher concentrate levels (Østergaard, 1979; Aston et al., 1995). Concentrate substitutes for silage, perhaps with increasing rates at high concentrate rations (Østergaard, 1979; Aston et al., 1995).

Glucogenic potential of diets based on restrictively fermented grass silage is quite low (Huhtanen, 1998). Glucose deficit leads to increased amino acid need for gluconeogenesis, thus limiting the amino acid supply to the mammary gland. Furthermore the glucose deficit increases the risk of ketosis. Subclinical ketosis, which is quite common during early lactation, prevents cows from achieving their potential yield and may decrease fertility (Andersson, 1988). Propylene glycol has been shown to increase blood glucose concentrations and it is widely used in prevention and treatment of ketosis (Emery et al., 1964; Sauer et al., 1973). Niacin has also elevated blood glucose and decreased $\beta$-hydroxybutyrate and NEFA (Dufva et al., 1983).

Our hypotheses were: 1 . Increased concentrate intake during early lactation increases milk and protein yields and decreases ad libitum grass silage intake and lipid mobilization. 2. Glucogenic supplement containing propylene glycol and niacin increases blood glucose concentration and decreases ketone concentrations and lipid mobilization.

\section{MATERIAL AND METHODS}

\section{Animals and experimental design}

Sixteen primiparous and sixteen multiparous dairy cows (Finnish Friesian) were divided to four treatment groups using a randomized complete block design. Four animal blocks were formed separately for primiparous and multiparous cows taking into account the expected calving date and liveweight for both parities and 
the milk yield of previous lactation and previous peak yicld for multiparous cows. The animals within the blocks were then allotted to the treatments.

The treatments were $2 \times 2$ factorially concentrate level and glucogenic liquid feed $(0$ or $1 \mathrm{l} / \mathrm{d}$; G0 or G1). Concentrate levels were $11 \mathrm{~kg} / \mathrm{d}$ and $15 \mathrm{~kg} / \mathrm{d}$ for multiparous cows and $9 \mathrm{~kg} / \mathrm{d}$ and $12 \mathrm{~kg} / \mathrm{d}$ for primiparous cows. Thus the treatments were $\mathrm{C} 11 \mathrm{G} 0, \mathrm{Cl1G} 1, \mathrm{C} 15 \mathrm{G} 0$ and $\mathrm{C} 15 \mathrm{Gl}$ for multiparous cows and $\mathrm{C} 9 \mathrm{G} 0, \mathrm{C} 9 \mathrm{G} 1$, $\mathrm{C} 12 \mathrm{G} 0$ and $\mathrm{C} 12 \mathrm{G} 1$ for primiparous cows.

The cows were kept in stalls and milked twice daily in a milking parlour. The milk yield was recorded for cvery milking. Liveweights were measured on two consecutive days three weeks before expected calving date, two weeks after calving and then every two weeks. Condition scoring (scale 1-5, Edmonson et al., 1989) was done by one person through the experiment at the same time as weighing.

Three weeks before calving average condition score of multiparous cows was 3.8 and average liveweight was $678 \mathrm{~kg}$. Cows in C15 group had significantly higher $(\mathrm{P}<0.05)$ condition scores than cows in $\mathrm{C} 11$ group (4.1 vs 3.5$)$. Average condition score of primiparous cows was 3.8 and average liveweight was $591 \mathrm{~kg}$. Average milk yicld of multiparous cows during previous lactation ( $305 \mathrm{~d}$ ) was $7778 \mathrm{~kg}$ energy corrected milk (ECM).

Feeds and feeding

The concentrate consisted of, \%: barley 30 , sugar bect pulp 25, rapeseed cake 18.75 , oats 10 , wheat bran 9.25 , molasses 5 and minerals 2 . The glucogenic liquid feed consisted of, \%: propylene glycol 24.4 , polyols 25 , sugar beet molasses 25 , xylose molasses 25 and nicotinamide 0.6 .

Three weeks before calving the daily concentrate ration was $2 \mathrm{~kg} / \mathrm{d}$ and the ration was increased to $3 \mathrm{~kg} / \mathrm{d}$ by the expected calving date. In order to satisfy the energy requirements, wilted silage was fed restrictively. Energy requirements were calculated according to Finnish standards (Tuori et al., 1996).

The experimental period started from parturition and lasted for 12 weeks. After calving, the daily concentrate amount of $15 \mathrm{~kg} / \mathrm{d}$ was achicved within 16 days. For two days after parturition the daily increase was $2 \mathrm{~kg}$, for the next two days $1 \mathrm{~kg}$ and after that $0.5 \mathrm{~kg} / \mathrm{d}$. The concentrate was fed four times a day. Wilted silage was from 1 st or 2 nd cut and it was given ad libitum, allowing 5-10\% refusals daily. The silage was fed twice a day. The concentrate and the silage were distributed with automated feeding cars. Feeds and refusals were weighed daily.

Because the crude protein content of the silage was lower than expected, rapeseed meal (RSM) was substituted for $6.67 \%$ of the concentrate. Glucogenic fecd ( 11 , specific weight $1.22 \mathrm{~kg} / \mathrm{l}$ ) was substituted for $1 \mathrm{~kg}$ concentrate in G1 groups. It was fed twice daily on top of the concentrate. Mineral supplement was given 
$100 \mathrm{~g} / \mathrm{d}$ to multiparous cows and $50 \mathrm{~g} / \mathrm{d}$ to primiparous cows at lower concentrate level when milk yield exceeded $25 \mathrm{~kg} / \mathrm{d}$.

Sampling and chemical analysis

One sample of concentrate and RSM was taken per batch. Silage was sampled weekly. Silage samples from the same silo were pooled although not for a longer period than one month. One sample was taken from the glucogenic feed.

Samples were analysed as described by Kokkonen et al. (2000). The gross encrgy of glucogenic feed was measured using a bomb calorimeter. Digestibility of silage was determined with four rams. During $21 \mathrm{~d}$ digestibility trial, the rams were placed in metabolism crates. Silage was fed at maintenance level. Faeces were collected daily during the last seven days of digestibility trial.

Milk samples were taken on four consecutive milkings starting two weeks after parturition and every two weeks thereafter. Fat, protein, lactose and urea contents of milk were determined as described by Kokkonen et al. (2000) and acetone content of milk with the methods described by Rajamäki and Rauramaa (1985). Milk samples for progesterone determination were taken after milking three times a weck starting 10 days after calving until first visible oestrus and stored frozen until determination with radioimmunoassay (RIA) (Laitinen, 1982). When the progesterone concentration exceeded $5 \mathrm{nmol} / \mathrm{l}$ at two consecutive samplings, the time of ovulation was estimated to be five days carlier than the rise of concentration. Due to missing samples only 23 animals were included for progesterone analysis.

Blood samples from the jugular vein were taken one weck before the expected calving day, and 2, 4 and 6 wecks after calving. Samples were taken to EDTA tubes before afternoon feeding at 13 o'clock. Blood samples for the determination of glucagon and insulin were immediately mixed with trypsin inhibitor (Aprotinin, Sigma A-1153). Plasma was separated by centrifuging and frozen until assay. Blood samples for the determination of $\beta$-hydroxybutyrate (BHB) and acetoacetate werc treated with $0.6 \mathrm{M}$ perchloric acid and frozen until analyses (Työppönen and Kauppinen, 1980). Glucose was determined enzymatically (Trinder, 1969). Insulin and glucagon were determined with RIA according to a kit procedure (Diagnostic Products Corporation, Coat-A-Count for insulin and Double Antibody Glucagon for glucagon). Non-esterified fatty acids (NEFA) (Wako Chemicals GmbH with the modifications by McCutcheon and Bauman, 1986) and triglycerides (Wahlefeld, 1974) were determined from plasma.

Calculations and statistical analysis

The Finnish AAT - PBV system is a modification of the Nordic AAT - PBV system (Tuori, 1992). AAT measures amino acids (bypass protein and microbial 
protein) absorbable from the small intestine. PBV (protein balance value) reflects the abundance of rumen degradable protein for rumen microbial synthesis. Microbial protein synthesis is proportional to the sum of digestible carbohydrates and rumen degradable protein. Effective protein degradability (EPD) value of $85 \%$ was used for silage.

A regression curve was fitted to the liveweight data of each animal to calculate liveweight changes. Energy corrected milk yield was calculated according to Sjaunja et al. (1990). Feeding values of concentrate and RSM were calculated using digestibilities from feed tables (Tuori et al., 1996). Feeding values of silages were calculated using digestibilities determined with sheep. Organic matter digestibility was $64 \%$ for 1 st cut silage and $78 \%$ for 2 nd cut silage.

For calculation of results (except blood sample data), the experiment was divided to two periods: period 1:1-42 d and period 2:43-84 d. Nutritional composition of feeds was calculated using the feed analyses for each period. Statistical analyses were performed using the GLM procedure of SAS (1989). The results of primiparous and multiparous cows were analysed separately. Milk yield, milk composition, feed intake, energy and nutrient intakes and utilisation, liveweight and liveweight changes and condition score were analysed according to the model:

$Y_{i, j}=\mu+C_{i}+G_{j}+B_{k}+e_{i j k}$ where $Y_{i j k}$ is the observation, $\mu$ is the overall mean, $C_{1}$ is the effect of concentrate level, $G_{j}$ is the effect of glucogenic feed, $B_{k}$ is the effect of block and $e_{i j k}$ is the residual effect.

Blood sample data were analysed within sampling time using the model above. If the prepartum sample was taken $2 \mathrm{~d}$ before calving or later, those animals were not used for prepartum blood data analyses. The BHB, acetoacetate, insulin and glucagon data were transformed to logarithms. Effects were considered to be different at $\mathrm{P}<0.05$, and tendencies were declared at $\mathrm{P}<0.10$.

\section{RESULTS}

Feed and diet composition and characteristics

The average chemical composition of the feeds is shown in Table 1. The fermentation quality of silages fed during the experiment was good. However there was considerable variation in chemical composition. Despite RSM supplementation the average crude protein (CP) concentration of diets was as low as $143 \mathrm{~g} \mathrm{CP} /$ $\mathrm{kg}$ DM. The diets contained on average $372 \mathrm{~g} \mathrm{NDF} / \mathrm{kg}$ DM and $200 \mathrm{~g} \mathrm{ADF} / \mathrm{kg}$ DM. The proportion of concentrate was 48 and $56 \%$ (DM basis) of the diet of multiparous cows with lower and higher concentrate levels and 52 and $56 \%$ of the diet of primiparous cows. 
TABLE 1

Chemical composition of feeds and feed values

\begin{tabular}{|c|c|c|c|c|c|}
\hline & & Silage & Concentrate & RSM & $\begin{array}{l}\text { Glucogenic } \\
\text { feed }\end{array}$ \\
\hline DM, g/kg & 277 & $(233-354)$ & 887 & 879 & 472 \\
\hline Ash, $\mathrm{g} / \mathrm{kg} \mathrm{DM}$ & 79 & $(71-96)$ & 76 & 78 & 71 \\
\hline $\mathrm{CP}, \mathrm{g} / \mathrm{kg} \mathrm{DM}$ & 112 & $(102-125)$ & 160 & 374 & 81 \\
\hline $\mathrm{EE}, \mathrm{g} / \mathrm{kg} \mathrm{DM}$ & 36 & $(31-44)$ & 49 & 55 & \\
\hline $\mathrm{CF}, \mathrm{g} / \mathrm{kg} \mathrm{DM}$ & 292 & $(219-328)$ & 99 & 119 & \\
\hline NDF, g/kg DM & 523 & $(419-582)$ & 261 & 264 & \\
\hline $\mathrm{ME}^{\prime}, \mathrm{MJ} / \mathrm{kg} \mathrm{DM}$ & 10.1 & $(9.4-11.4)$ & 12.5 & 11.7 & $19.0^{!}$ \\
\hline$\wedge \wedge \mathrm{T}^{2}, \mathrm{~g} / \mathrm{kg} \mathrm{DM}$ & 78 & $(72-87)$ & 109 & 165 & 97 \\
\hline $\mathrm{PBV}^{2}, \mathrm{~g} / \mathrm{kg} \mathrm{DM}$ & -21 & $(-44 \ldots-6)$ & -15 & 123 & $-8 !$ \\
\hline $\mathrm{pH}$ & \multicolumn{2}{|c|}{4.03} & & & \\
\hline Lactic acid, $\mathrm{g} / \mathrm{kg}$ DM & 67 & $(10-107)$ & & & \\
\hline Acetic acid, $\mathrm{g} / \mathrm{kg} \mathrm{DM}$ & 15 & $(4-24)$ & & & 8.4 \\
\hline Propionic acid, g/kg DM & 0.6 & $(0.1-1.3)$ & & & 0.4 \\
\hline Butyric acid, g/kg DM & 0.3 & $(0.1-0.7)$ & & & \\
\hline Isobutyric acid. $\mathrm{g} / \mathrm{kg}$ DM & \multicolumn{2}{|c|}{0.08} & 1.1 & & \\
\hline Sugars, g/kg DM & 92 & $(18-221)$ & & & 465 \\
\hline Soluble N, g/kg N & 539 & $(451-604)$ & & & \\
\hline $\mathrm{NH}_{3}-\mathrm{N}, \mathrm{g} / \mathrm{kg} \mathrm{N}$ & 44 & $(32-55)$ & & & \\
\hline
\end{tabular}

$1 \mathrm{ME}=\mathrm{GE} \times$ digestibility $\times \mathrm{ME} / \mathrm{DE}$, where digestibility $=0.95$ and $\mathrm{ME} / \mathrm{DE}=0.84$

2 Finnish AAT - PBV protein evaluation system is a modification of the Nordic AAT . PBV system. see Tuori et al. (1996)

\section{Multiparous cows}

There was a significant interaction $(\mathrm{P}<0.01$ and $\mathrm{P}<0.05)$ in silage dry matter intake (DMI) between concentrate level and glucogenic feed in both periods (Tables 2 and 3$)$ and in total DMI $(\mathrm{P}<0.05)$ during period 1 . Without glucogenic feed silage DMI was lower at the higher level of concentrate, but there was only a marginal effect with glucogenic feed. Total DMI was higher $(\mathrm{P}<0.01$ and $\mathrm{P}<0.001)$ in $\mathrm{C} 15$ groups in both periods. Difference of total DMI was greater between C15G1 and $\mathrm{C} 11 \mathrm{Gl}$ than between the two G0 groups. This interaction was significant $(\mathrm{P}<0.05)$ during period 1. ME and AAT intakes were higher $(\mathrm{P}<0.01$ and $\mathrm{P}<0.001)$ with the higher concentrate level in both periods. The same kind of interaction $(\mathrm{P}<0.10$ or better) was seen in these parameters as in total DMI during period 1 . ME intake was higher $(\mathrm{P}<0.05)$ and $\mathrm{PBV}$ was lower $(\mathrm{P}<0.05)$ with glucogenic feed during period 2.

There was a significant interaction $(\mathrm{P}<0.05)$ in milk yield between concentrate level and glucogenic feed during period 1. Milk yield was higher with 
TABLE 2

Feed intake, milk yield and feed utilisation, multiparous cows (lactation days 1-42)

\begin{tabular}{|c|c|c|c|c|c|c|c|}
\hline & \multicolumn{4}{|c|}{ Treatments } & \multirow{2}{*}{ SEM } & \multicolumn{2}{|c|}{ Significance } \\
\hline & C.1160 & $\mathrm{C}[1 \mathrm{G} 1$ & $\mathrm{C} 15 \mathrm{G} 0$ & $\mathrm{C} 15 \mathrm{Gl}$ & & C $\mathrm{G}$ & $\mathrm{C} \times \mathrm{G}$ \\
\hline \multicolumn{8}{|l|}{ Fced intake, kg DM/d } \\
\hline silage & 10.83 & 9.36 & 8.61 & 9.28 & 0.313 & $* *$ & $* *$ \\
\hline concentrate & 9.06 & 9.28 & 11.58 & 11.73 & 0.099 & $* * *$ & \\
\hline total $^{1}$ & 19.89 & 18.64 & 20.19 & 21.00 & 0.361 & $* *$ & * \\
\hline $\mathrm{ME}, \mathrm{MJ} / \mathrm{d}$ & 225 & 217 & 231 & 245 & 4.93 & $* *$ & o \\
\hline$C P, g / d$ & 2840 & 2595 & 2963 & 3035 & 45.6 & $* * *$ & $* *$ \\
\hline$\wedge \Lambda \mathrm{T}, \mathrm{g} / \mathrm{d}$ & 1883 & 1763 & 1965 & 2028 & 35.7 & $* *$ & $*$ \\
\hline $\mathrm{PBV}, \mathrm{g} / \mathrm{d}$ & -259 & -305 & -250 & -287 & 49.8 & & \\
\hline $\mathrm{CF}, \mathrm{g} / \mathrm{d}$ & 3993 & 3543 & 3642 & 3845 & 119.7 & & $*$ \\
\hline $\mathrm{NDF}, \mathrm{g} / \mathrm{d}$ & 8017 & 7178 & 7683 & 7869 & 217.3 & & * \\
\hline$A D F, g / d$ & 4346 & 3861 & 4023 & 4217 & 123.5 & & * \\
\hline \multicolumn{8}{|c|}{ Milk and milk component yicld. $\mathrm{kg} / \mathrm{d}$} \\
\hline milk & 36.73 & 31.69 & 36.62 & 38.00 & 1.08 & * & $*$ \\
\hline $\mathrm{ECM}$ & 35.55 & 31.52 & 34.55 & 36.45 & 1.18 & & $*$ \\
\hline fat yield & 1.38 & 1.24 & 1.28 & 1.39 & 0.075 & & \\
\hline protein yield & 1.16 & 1.05 & 1.21 & 1.22 & $0.04 \mathrm{I}$ & $*$ & \\
\hline lactose yield & 1.80 & 1.53 & 1.79 & 1.88 & 0.064 & * & $*$ \\
\hline \multicolumn{8}{|l|}{ Milk composition, $\mathrm{g} / \mathrm{kg}$} \\
\hline fat & 38.0 & 38.8 & 34.9 & 37.0 & 2.27 & & \\
\hline protein & 31.7 & 33.3 & 33.1 & 32.1 & 0.895 & & \\
\hline lactose & 49.0 & 48.0 & 48.8 & 49.5 & 1.06 & & \\
\hline urea, $\mathrm{mg} / 100 \mathrm{ml}$ & 14.3 & 17.0 & 18.2 & 17.6 & 1.87 & & \\
\hline \multicolumn{8}{|c|}{ Feed utilisation and liveweight change } \\
\hline LWC, $\mathrm{kg} / \mathrm{d}$ & 0.34 & -0.14 & -1.28 & -0.88 & 0.560 & $\circ$ & \\
\hline condition score & 3.0 & 2.9 & 3.2 & 3.4 & 0.17 & $\circ$ & \\
\hline $\mathrm{k}_{\mathrm{i}}^{2}$ & 0.74 & 0.65 & 0.56 & 0.58 & 0.053 & * & \\
\hline milk protcin $/ \mathrm{CP}, \mathrm{g} / \mathrm{g}$ & 0.41 & 0.40 & 0.41 & 0.40 & 0.014 & & \\
\hline
\end{tabular}

1 includes mineral supplement in lower concentrate level

$2 \mathrm{k}_{1}=\mathrm{ECM} \times 3.14 /(\mathrm{ME}$ intake $-\mathrm{ME}$ allowance for maintenance $\cdots \mathrm{ME}$ of LWC $\mathrm{LWW}$ ), where ME of LWC is $28 \mathrm{MJ} / \mathrm{kg} \mathrm{LW}$ loss and $34 \mathrm{MJ} / \mathrm{kg} \mathrm{LW}$ gain and $\mathrm{ME}$ allowance for maintenance is calculated according to MAFF (1975)

$\mathrm{ECM}=$ energy corrected milk yield; $\mathrm{k}_{1}=\mathrm{ME}$ utilisation for milk production ${ }^{\circ} \mathrm{P}<0.10{ }^{*} \mathrm{P}<0.05 ; * * \mathrm{P}<0.01 ;{ }^{* *} \mathrm{P}<0.001 ; \mathrm{C}=$ concentrate, $\mathrm{GF}=$ glucogenic feed 
TABLLE: 3

Feed intake, milk yicld and feed utilisation, multiparous cows (lactation days 43 - 84)

\begin{tabular}{|c|c|c|c|c|c|c|c|c|}
\hline & \multicolumn{4}{|c|}{ Treatments } & \multirow{2}{*}{ SEM } & \multicolumn{3}{|c|}{ Significance } \\
\hline & $\mathrm{Cl} 1 \mathrm{G} 0$ & $\mathrm{CllGl}$ & C15G0 & $\mathrm{C} 15 \mathrm{G} 1$ & & $\mathrm{C}$ & $\mathrm{G}$ & $\mathrm{C} \times \mathrm{G}$ \\
\hline \multicolumn{9}{|l|}{ Feed intake. kg DM/d } \\
\hline silage & 10.74 & 10.36 & 9.20 & 10.82 & 0.403 & & & * \\
\hline concentrate & 9.60 & 9.64 & 13.04 & 13.03 & 0.042 & $* * *$ & & \\
\hline total' & 20.34 & 20.00 & 22.24 & 23.85 & 0.043 & $* * *$ & $*$ & \\
\hline $\mathrm{ME}, \mathrm{MJ} / \mathrm{d}$ & 231 & 237 & 263 & 288 & 5.28 & $* * *$ & $*$ & \\
\hline $\mathrm{CP}, \mathrm{g} / \mathrm{d}$ & 2914 & 2781 & 3322 & 3419 & 45.5 & $* * *$ & & $*$ \\
\hline AAT, g/d & 1946 & 1927 & 2237 & 2362 & 39.3 & $* * *$ & & \\
\hline $\mathrm{PBV}, \mathrm{g} / \mathrm{d}$ & -284 & -391 & -333 & -452 & 38.9 & & * & \\
\hline $\mathrm{CF}, \mathrm{g} / \mathrm{d}$ & 3918 & 3581 & 3645 & 3927 & 143 & & & $\circ$ \\
\hline$N D F, g / d$ & 7977 & 7331 & 7794 & 8274 & 248 & & & * \\
\hline ADF, $g / d$ & 4304 & 3945 & 4071 & 4370 & 150 & & & $\circ$ \\
\hline \multicolumn{9}{|c|}{ Milk and milk component yield, $\mathrm{kg} / \mathrm{d}$} \\
\hline milk & 39.00 & 34.27 & 38.51 & 38.95 & 1.91 & & & \\
\hline $\mathrm{ECM}$ & 35.14 & 32.57 & 37.06 & 35.28 & 1.46 & & & \\
\hline fat yield & 1.30 & 1.24 & 1.41 & 1.27 & 0.070 & & & \\
\hline protein yicld & 1.14 & 1.09 & 1.25 & 1.18 & 0.042 & $*$ & & \\
\hline lactose yield & 1.94 & 1.68 & 1.90 & 1.98 & 0.099 & & & \\
\hline \multicolumn{9}{|l|}{ Milk composition, $\mathrm{g} / \mathrm{kg}$} \\
\hline fat & 33.6 & 35.9 & 36.8 & 32.8 & 1.91 & & & \\
\hline protein & 29.3 & 31.8 & 32.8 & 30.4 & 0.672 & & & $* *$ \\
\hline lactose & 49.8 & 49.0 & 49.4 & 50.8 & 0.932 & & & \\
\hline urea, $\mathrm{mg} / 100 \mathrm{ml}$ & 19.8 & 15.2 & 17.2 & 17.3 & 1.46 & & & \\
\hline \multicolumn{9}{|c|}{ Feed utilisation and liveweight change } \\
\hline LWC, $\mathrm{kg} / \mathrm{d}$ & -0.06 & 0.54 & 0.34 & 0.28 & 0.229 & & & \\
\hline condition score & 2.9 & 3.0 & 3.2 & 3.3 & 0.13 & $\circ$ & & \\
\hline $\mathrm{k}_{\mathrm{i}}^{-}$ & 0.65 & 0.66 & 0.63 & 0.52 & 0.029 & * & $\circ$ & $\circ$ \\
\hline milk protein/CP, $\mathrm{g} / \mathrm{g}$ & 0.39 & 0.39 & 0.38 & 0.34 & 0.017 & & & \\
\hline
\end{tabular}

1 includes mineral supplement in lower concentrate level

${ }^{2} \mathrm{k}_{1}=\mathrm{ECM} \times 3.14$ / (ME intake - ME allowance for maintenance -- ME of LWC x LWC), where ME of LWC is $28 \mathrm{MJ} / \mathrm{kg} \mathrm{LW}$ loss and $34 \mathrm{MJ} / \mathrm{kg} \mathrm{LW}$ gain and ME allowance for maintenance is calculated according to MAFF (1975)

$\mathrm{ECM}=$ energy corrected milk yield; $\mathrm{k}_{1}=\mathrm{ME}$ utilisation for milk production

${ }^{\circ} \mathrm{P}<0.10 ;{ }^{*} \mathrm{P}<0.05 ; * * \mathrm{P}<0.01 ; * * * \mathrm{P}<0.001$ 
glucogenic feed at the higher concentrate level whereas the opposite was true at the lower concentrate level. Milk, protein and lactose yields were higher $(\mathrm{P}<0.05)$ in $\mathrm{C} 15$ groups during period 1. Only protein yicld was higher $(\mathrm{P}<0.05)$ at the higher level of concentrate during period 2. There were significant $(\mathrm{P}<0.05$ or better) interactions between concentrate level and glucogenic feed in ECM and lactose yields during period 1 and in protein concentration of milk during pcriod 2. ME utilisation for milk production $\left(\mathrm{k}_{1}\right)$ was higher $(\mathrm{P}<0.05)$ in $\mathrm{C} 11$ groups in both periods.

Plasma glucagon concentrations were higher $(\mathrm{P}<0.10$ or better $)$ in $\mathrm{C} 15$ groups in all post partum samplings (Table 6 ). Glucose concentration tended to bc higher $(\mathrm{P}<0.10)$ with glucogenic feed at 2 and 6 weeks post partum. At the same time BHB and acetoacetate concentrations were lower $(\mathrm{P}<0.10$ or better $)$ with glucogenic feed.

According to progesterone concentrations, the time of first ovulation was 37 , 32.12 and 24 days from parturition in groups $\mathrm{C} 11 \mathrm{G} 0, \mathrm{C} 11 \mathrm{G} 1, \mathrm{C} 15 \mathrm{G} 0$ and $\mathrm{C} 15 \mathrm{G} 1$. The number of open days was $79,108,89$ and 74 . There was no difference in incidences of diseases (data not shown).

\section{Primiparous cows}

Neither daily concentrate ration nor glucogenic feed had a significant effect on silage DM intake (Tables 4 and 5). Total DM intake, ME intake and CP and AAT intakes were higher ( $\mathrm{P}<0.01$ or better) in $\mathrm{C} 12$ groups during both periods.

There were no significant $(\mathrm{P}<0.05)$ effects on milk yield or milk composition. Liveweight gain tended to be higher $(\mathrm{P}<0.10)$ with the higher concentrate ration during period 2. Encrgy ( $\mathrm{P}<0.10$ or better) and protein ( $\mathrm{g}$ milk protein/g CP) $(\mathrm{P}<0.05$ or better) utilisation were lower in both periods in $\mathrm{C} 12$ groups. Protein utilisation tended to be higher $(\mathrm{P}<0.10)$ with glucogenic feed in both periods.

Plasma glucose concentrations were higher $(\mathrm{P}<0.05)$ in $\mathrm{C} 12$ groups 4 weeks post partum, and $\mathrm{BHB}$ and acetoacetate concentrations were lower $(\mathrm{P}<0.01)$ 6 wecks post partum (Table 7). Insulin concentrations tended to be higher $(\mathrm{P}<0.10)$ 6 weeks post partum in $\mathrm{C} 12$ groups. Triglyceride concentrations 2 weeks post partum were lower $(\mathrm{P}<0.05)$ with glucogenic feed, and BHB and glucagon concentration tended to be lower $(\mathrm{P}<0.10) 6$ weeks after calving with glucogenic feed.

According to progesterone concentrations, the time of first ovulation was 43 , 31,33 and 36 days from parturition in groups C9G0, C9G1, C12G0 and C12G1. The number of open days was $129,86,95$ and 90 . The only clinically ketotic cow in the experiment was in group $\mathrm{C} 9 \mathrm{GO}$. 
TABLE 4

Feed intake. milk yield and feed utilisation, primiparous cows (lactation days 1-42)

\begin{tabular}{|c|c|c|c|c|c|c|c|c|}
\hline & \multicolumn{4}{|c|}{ Treatments } & \multirow{2}{*}{ SEM } & \multicolumn{3}{|c|}{ Significance } \\
\hline & $\mathrm{C9G0}$ & $\mathrm{C} 9 \mathrm{Gl}$ & $\mathrm{Cl} 2 \mathrm{G} 0$ & $\mathrm{C} 12 \mathrm{G} 1$ & & $\mathrm{C}$ & $\mathrm{G}$ & $\mathrm{CxG}$ \\
\hline \multicolumn{9}{|c|}{ Feed intake, $\mathrm{kg} \mathrm{DM} / \mathrm{d}$} \\
\hline silage & 7.46 & 6.17 & 7.58 & 6.73 & 0.736 & & & \\
\hline concentrate & 7.47 & 7.66 & 9.66 & 9.89 & 0.060 & $* * *$ & $* *$ & \\
\hline total' & 14.93 & 13.83 & 17.24 & 16.62 & 0.741 & $* *$ & & \\
\hline $\mathrm{ME}, \mathrm{MJ} / \mathrm{d}$ & 172 & 166 & 202 & 202 & 7.61 & $* *$ & & \\
\hline CP. $g / d$ & 2144 & 1948 & 2501 & 2362 & 94.1 & $* *$ & & \\
\hline $\mathrm{AAT}, \mathrm{g} / \mathrm{d}$ & 14.38 & 1341 & 1700 & 1648 & 60.9 & $* *$ & & \\
\hline $\mathrm{PBV}, \mathrm{g} / \mathrm{d}$ & -221 & -256 & -287 & -338 & 33.5 & $\circ$ & & \\
\hline$C F, g / d$ & $272 !$ & 2344 & 2895 & 2604 & 224.6 & & & \\
\hline$N D F, g / d$ & 5721 & 4898 & 6225 & 5592 & 418.8 & & & \\
\hline $\mathrm{ADF}, \mathrm{g} / \mathrm{d}$ & 2984 & 2580 & 3204 & 2884 & 242.1 & & & \\
\hline
\end{tabular}

Milk and milk component yield, $\mathrm{kg} / \mathrm{d}$

$\begin{array}{lrrrrl}\text { milk } & 24.94 & 27.94 & 27.35 & 27.04 & 1.17 \\ \text { ECM } & 24.90 & 26.10 & 25.85 & 27.04 & 0.900 \\ \text { fat yield } & 0.98 & 0.98 & 0.95 & 1.05 & 0.043 \\ \text { protein yield } & 0.83 & 0.86 & 0.87 & 0.88 & 0.034 \\ \text { lactose yield } & 1.23 & 1.40 & 1.39 & 1.37 & 0.063\end{array}$

Milk composition, $\mathrm{g} / \mathrm{kg}$

$\begin{array}{llllll}\text { fat } & 39.2 & 35.3 & 35.0 & 39.4 & 1.98 \\ \text { protein } & 33.2 & 30.8 & 32.1 & 32.7 & 0.839 \\ \text { lactose } & 49.1 & 50.0 & 50.6 & 50.7 & 0.743 \\ \text { urea, } \mathrm{mg} / 100 \mathrm{~m} ! & 16.2 & 13.2 & 15.1 & 13.2 & 2.67\end{array}$

Feed utilisation and liveweight change

$\begin{array}{lccccccc}\mathrm{LWC}, \mathrm{kg} / \mathrm{d} & -0.45 & -0.66 & -0.55 & -0.13 & 0.191 & & \\ \text { condition score } & 3.5 & 3.1 & 3.2 & 3.2 & 0.18 & & \\ \mathrm{k}_{\mathrm{b}}{ }^{2} & 0.65 & 0.64 & 0.50 & 0.61 & 0.045 & \circ & \\ \text { milk protein } / \mathrm{Cl}^{3}, \mathrm{~g} / \mathrm{g} & 0.39 & 0.44 & 0.35 & 0.37 & 0.018 & * & *\end{array}$

1 includes mineral supplement in lower concentrate level

$2 \mathrm{k}_{\mathrm{t}}=\mathrm{ECM} \times 3.14$ / (ME intake - ME allowance for maintenance- ME of I.WC x LWC), where ME of LWC is $28 \mathrm{MJ} / \mathrm{kg} \mathrm{LW}$ loss and $34 \mathrm{MJ} / \mathrm{kg} \mathrm{LW}$ gain and ME allowance for maintenance is calculated according to MAFF (1975)

$\mathrm{ECM}=$ energy corrected milk yield; $\mathrm{k}_{1}=\mathrm{ME}$ utilisation for milk production

${ }^{\circ} \mathrm{P}<0.10 ; * \mathrm{P}<0.05 ;{ }^{* *} \mathrm{P}<0.01 ; * * * \mathrm{P}<0.001$ 
TABLE 5

Feed intake. milk yield and feed utilisation, primiparous cows (lactation days 43-84)

\begin{tabular}{|c|c|c|c|c|c|c|c|c|}
\hline & \multicolumn{4}{|c|}{ Treatments } & \multirow{2}{*}{ SEM } & \multicolumn{3}{|c|}{ Significance } \\
\hline & $\mathrm{C} 9 \mathrm{G} 0$ & C9Gl & $\mathrm{C} 12 \mathrm{G} 0$ & $\mathrm{C} 12 \mathrm{Gl}$ & & $\mathrm{C}$ & $\mathrm{G}$ & $\mathrm{C} \times \mathrm{G}$ \\
\hline \multicolumn{9}{|l|}{ Feed intake, $\mathrm{kg} \mathrm{DM} / \mathrm{d}$} \\
\hline silage & 8.54 & 7.26 & 8.79 & 8.85 & 0.791 & & & \\
\hline concentrate & 7.78 & 7.95 & 10.49 & 10.59 & 0.069 & $* * *$ & $\circ$ & \\
\hline total' & 16.32 & 15.21 & 19.28 & 19.43 & 0.826 & $* *$ & & \\
\hline $\mathrm{ME}, \mathrm{MJ} / \mathrm{d}$ & 184 & 177 & 220 & 228 & 9.86 & $* *$ & & \\
\hline $\mathrm{CP}, \mathrm{g} / \mathrm{d}$ & 2342 & 2133 & 2866 & 2796 & 96.8 & $* * *$ & & \\
\hline $\mathrm{AAT}, \mathrm{g} / \mathrm{d}$ & 1543 & 1436 & 1872 & 1877 & 75.4 & $* *$ & & \\
\hline $\mathrm{PBV}, \mathrm{g} / \mathrm{d}$ & -194 & -225 & -199 & -283 & 49.4 & & & \\
\hline $\mathrm{CF}, \mathrm{g} / \mathrm{d}$ & 3302 & 2932 & 3609 & 3513 & 236.6 & $\circ$ & & \\
\hline NDF, $g / d$ & 6588 & 5841 & 7399 & 7148 & 419.3 & $*$ & & \\
\hline $\mathrm{ADF}, \mathrm{g} / \mathrm{d}$ & 3600 & 3201 & 3971 & 3873 & 247.1 & $\circ$ & & \\
\hline \multicolumn{9}{|c|}{ Milk and milk component yield, $\mathrm{kg} / \mathrm{d}$} \\
\hline milk & 26.78 & 29.42 & 29.40 & 29.57 & 1.38 & & & \\
\hline ECM & 26.76 & 27.59 & 27.07 & 28.11 & 0.965 & & & \\
\hline fat yicld & 1.06 & 1.05 & 1.01 & 1.04 & 0.038 & & & \\
\hline protein yield & 0.87 & 0.89 & 0.88 & 0.94 & 0.033 & & & \\
\hline lactose yield & 1.32 & 1.47 & 1.48 & 1.53 & 0.074 & & & \\
\hline \multicolumn{9}{|l|}{ Milk composition. $\mathrm{g} / \mathrm{kg}$} \\
\hline fat & 39.9 & 36.0 & 34.5 & 35.5 & 1.68 & & & \\
\hline protcin & 32.4 & 30.5 & 30.0 & 31.7 & 0.822 & & & $\circ$ \\
\hline lactose & 49.3 & 50.1 & 50.3 & 51.9 & 0.729 & $\circ$ & & \\
\hline urea, $\mathrm{mg} / 100 \mathrm{ml}$ & 19.4 & 17.7 & 20.3 & 18.3 & 2.47 & & & \\
\hline \multicolumn{9}{|c|}{ Feed utilisation and liveweight change } \\
\hline $\mathrm{LWC}, \mathrm{kg} / \mathrm{d}$ & 0.34 & 0.18 & 0.48 & 0.61 & 0.129 & $\circ$ & & \\
\hline condition score & 3.6 & 3.1 & 3.3 & 3.3 & 0.18 & & & \\
\hline $\mathrm{MJ} / \mathrm{kg} \mathrm{ECM}$ & 4.2 & 4.2 & 5.4 & 5.2 & 0.40 & * & & \\
\hline $\mathrm{k}_{\mathrm{l}}^{2}$ & 0.74 & 0.78 & 0.58 & 0.62 & 0.061 & * & & \\
\hline milk protein $/ \mathrm{CP}, \mathrm{g} / \mathrm{g}$ & 0.37 & 0.42 & 0.31 & 0.34 & 0.019 & $* *$ & $\circ$ & \\
\hline
\end{tabular}

includes mineral supplement in lower concentrate level

$\therefore \mathrm{k}_{1}=\mathrm{ECM} \times 3.14 /$ (ME intake $\cdots$ ME allowance for maintenance -- ME of LWC $\times$ LWC), where ME of LWC is $28 \mathrm{MJ} / \mathrm{kg} \mathrm{LW}$ loss and $34 \mathrm{MJ} / \mathrm{kg} \mathrm{LW}$ gain and ME allowance for maintenance is calculated according to MAFF (1975)

$\mathrm{ECM}=$ energy corrected milk yield; $\mathrm{k}_{1}=\mathrm{ME}$ utilisation for milk production

${ }^{\circ} \mathrm{P}<0.10 ;{ }^{*} \mathrm{P}<0.05 ; * * \mathrm{P}<0.01 ; * * * \mathrm{P}<0.001$ 
TABILE 6

Blood composition, multiparous cows

\begin{tabular}{|c|c|c|c|c|c|c|c|c|}
\hline & \multicolumn{4}{|c|}{ Treatments } & \multirow{2}{*}{ SE } & \multicolumn{3}{|c|}{ Significance } \\
\hline & $\mathrm{CI}$ GO & $\mathrm{C} 11 \mathrm{G} 1$ & $\mathrm{C} 15 \mathrm{GO}$ & $\mathrm{C} 15 \mathrm{G} 1$ & & $\mathrm{C}$ & $\mathrm{G}$ & $\mathrm{C} \times \mathrm{G}$ \\
\hline \multicolumn{9}{|l|}{ I week' } \\
\hline glucose, $\mathrm{mg} / 100 \mathrm{ml}$ & 61.6 & 58.8 & 66.7 & 62.9 & 3.49 & $*$ & & \\
\hline Nefa, mEq/l & 0.12 & 0.19 & 0.22 & 0.20 & 0.095 & & & \\
\hline BHB, $\mathrm{mmol} / \mathrm{l}$ & 0.34 & 0.36 & 0.31 & 0.32 & 0.047 & & & \\
\hline insulin $\mu \mathrm{JU} / \mathrm{mi}$ & 9.9 & 12.7 & 11.1 & 12.8 & 4.24 & & & \\
\hline triglyserides, $\mathrm{mmol} / \mathrm{t}$ & 0.42 & 0.34 & 0.42 & 0.50 & 0.087 & & & \\
\hline \multicolumn{9}{|l|}{2 weeks 23} \\
\hline glucose, $\mathrm{mg} / 100 \mathrm{ml}$ & 46.0 & 56.6 & 56.2 & 61.6 & 7.79 & & $\circ$ & \\
\hline Nefa, mEq/l & 0.50 & 0.41 & 0.47 & 0.67 & 0.27 & & & \\
\hline $\mathrm{BHB}, \mathrm{mmol} / 1$ & 1.11 & 0.49 & 1.01 & 0.39 & 0.66 & & $\circ$ & \\
\hline insulin $\mu \mathrm{IU} / \mathrm{ml}$ & 7.1 & 8.7 & 7.2 & 7.8 & 2.76 & & & \\
\hline triglyserides, mmol/1 & 0.30 & 0.18 & 0.24 & 0.27 & 0.097 & & & \\
\hline glucagon, $\mathrm{pg} / \mathrm{ml}$ & 66.3 & 49.9 & 82.5 & 84.9 & 20.6 & $\circ$ & & \\
\hline acetoacetate, $\mathrm{mmol} / \mathrm{l}$ & 0.19 & 0.03 & 0.12 & 0.00 & 0.18 & & $\circ$ & \\
\hline \multicolumn{9}{|l|}{4 weeks } \\
\hline glucose, $\mathrm{mg} / 100 \mathrm{ml}$ & 54.3 & 61.5 & 58.8 & 66.3 & 6.79 & & & \\
\hline Nefa, mEq/l & 0.31 & 0.33 & 0.38 & 0.36 & 0.18 & & & \\
\hline BHB. minol/l & 0.97 & 0.73 & 0.97 & 0.75 & 0.37 & & & \\
\hline insulin $\mu[\mathrm{U} / \mathrm{ml}$ & 8.7 & 10.0 & 9.4 & 9.8 & 2.87 & & & \\
\hline triglyserides, mmol/1 & 0.20 & 0.26 & 0.20 & 0.30 & 0.094 & & & \\
\hline glucagon, $\mathrm{pg} / \mathrm{ml}$ & 78.1 & 51.5 & 99.4 & 83.1 & 21.8 & $*$ & $\circ$ & \\
\hline acetoacetate, mol/l & 0.11 & 0.08 & 0.13 & 0.06 & 0.12 & & & \\
\hline \multicolumn{9}{|l|}{6 weeks } \\
\hline glucosc. $\mathrm{mg} / 100 \mathrm{ml}$ & 61.3 & 66.5 & 60.5 & 72.5 & 8.82 & & $\circ$ & \\
\hline Nefa, mEq/I & 0.28 & 0.25 & 0.32 & 0.27 & 0.13 & & & \\
\hline BHB. mmol/s & 1.69 & 0.52 & 0.97 & 0.49 & 0.42 & & $* *$ & \\
\hline insulin $\mu \mathrm{JU} / \mathrm{ml}$ & 8.1 & 11.6 & 9.5 & 12.7 & 3.35 & & 0 & \\
\hline triglyserides, mmol/l & 0.23 & 0.19 & 0.20 & 0.18 & 0.049 & & & \\
\hline glucagon, pg/ml & 76.3 & 58.6 & 96.7 & 91.6 & 23.4 & $*$ & & \\
\hline acctoacetate, mmol/l & 0.29 & 0.05 & 0.14 & 0.03 & 0.10 & & $\circ$ & \\
\hline
\end{tabular}

C $11 \mathrm{G} 0$ and $\mathrm{C} 11 \mathrm{G} 1: \mathrm{n}=3, \mathrm{C} 15 \mathrm{G} 0$ and $\mathrm{C} 15 \mathrm{Gl}: \mathrm{n}=4$

$=\mathrm{C} 15 \mathrm{Gl}: \mathrm{n}=3, \mathrm{C} 11 \mathrm{G} 0, \mathrm{C} 11 \mathrm{Gl}$ and $\mathrm{C} 15 \mathrm{G} 0: \mathrm{n}=4$

* as in Table 5 
TABLE 7

Blood composition, primiparous cows

\begin{tabular}{|c|c|c|c|c|c|c|c|c|}
\hline & \multicolumn{4}{|c|}{ Treatments } & \multirow{2}{*}{ SE } & \multicolumn{3}{|c|}{ Significance } \\
\hline & C9G0 & C9G1 & $\mathrm{C} 12 \mathrm{G} 0$ & $\mathrm{C} 12 \mathrm{G} 1$ & & $\mathrm{C}$ & $\mathrm{G}$ & $\mathrm{C} \times \mathrm{G}$ \\
\hline \multicolumn{9}{|l|}{-1 week' } \\
\hline glucose, $\mathrm{mg} / 100 \mathrm{ml}$ & 62.2 & 70.3 & 61.9 & 68.9 & 4.76 & & 。 & \\
\hline $\mathrm{Nefa}, \mathrm{mEq} / \mathrm{l}$ & 0.25 & 0.25 & 0.19 & 0.21 & 0.17 & & & \\
\hline $\mathrm{BHB}, \mathrm{mmol} / \mathrm{l}$ & 0.34 & 0.20 & 0.32 & 0.28 & 0.056 & & * & \\
\hline insulin $\mu \mathrm{IU} / \mathrm{ml}$ & 11.5 & 15.9 & 15.4 & 11.1 & 3.81 & & & \\
\hline triglyserides, $\mathrm{mmol} / \mathrm{l}$ & 0.44 & 0.44 & 0.39 & 0.43 & 0.087 & & & \\
\hline \multicolumn{9}{|l|}{2 weeks } \\
\hline glucose, $\mathrm{mg} / 100 \mathrm{ml}$ & 62.4 & 62.0 & 66.9 & 64.5 & 7.49 & & & \\
\hline Nefa, $\mathrm{mEq} / 1$ & 0.49 & 0.32 & 0.40 & 0.46 & 0.31 & & & \\
\hline $\mathrm{BHB}, \mathrm{mmol} / \mathrm{l}$ & 0.86 & 0.61 & 0.37 & 0.59 & 0.52 & & & \\
\hline insulin $\mu \mathrm{IU} / \mathrm{ml}$ & 11.5 & 9.4 & 9.9 & 9.3 & 3.87 & & & \\
\hline triglyserides, $\mathrm{mmol} / \mathrm{l}$ & 0.33 & 0.18 & 0.23 & 0.16 & 0.077 & & $*$ & \\
\hline glucagon, $\mathrm{pg} / \mathrm{ml}$ & 80.1 & 52.8 & 66.7 & 66.6 & 44.1 & & & \\
\hline acetoacetate, $\mathrm{mmol} / \mathrm{l}$ & 0.13 & 0.06 & 0.01 & 0.04 & 0.13 & & & \\
\hline \multicolumn{9}{|l|}{4 weeks } \\
\hline glucose, $\mathrm{mg} / 100 \mathrm{ml}$ & 59.9 & 63.9 & 68.6 & 72.9 & 6.40 & * & & \\
\hline $\mathrm{Nefa}, \mathrm{mEq} / \mathrm{l}$ & 0.33 & 0.22 & 0.25 & 0.25 & 0.18 & & & \\
\hline $\mathrm{BHB}, \mathrm{mmol} / \mathrm{l}$ & 1.09 & 0.66 & 0.71 & 0.39 & 0.60 & & & \\
\hline insulin $\mu \mathrm{IU} / \mathrm{ml}$ & 8.4 & 11.1 & 14.6 & 11.4 & 5.12 & & & \\
\hline triglyserides, $\mathrm{mmol} / 1$ & 0.21 & 0.26 & 0.20 & 0.19 & 0.064 & & & \\
\hline glucagon, pg/ml & 96.0 & 61.0 & 76.3 & 68.7 & 30.9 & & & \\
\hline acetoacetate, $\mathrm{mmol} / \mathrm{l}$ & 0.20 & 0.08 & 0.05 & 0.01 & 0.18 & & & \\
\hline \multicolumn{9}{|l|}{6 weeks } \\
\hline glucose, $\mathrm{mg} / 100 \mathrm{ml}$ & 60.1 & 67.3 & 68.1 & 71.3 & 7.39 & & & \\
\hline Nefa, $\mathrm{mEq} / \mathrm{l}$ & 0.26 & 0.27 & 0.26 & 0.22 & 0.18 & & & \\
\hline $\mathrm{BHB}, \mathrm{mmol} / \mathrm{l}$ & 2.01 & 0.64 & 0.42 & 0.34 & 0.89 & $* *$ & $\circ$ & \\
\hline insulin $\mu \mathrm{IU} / \mathrm{ml}$ & 6.8 & 9.7 & 11.0 & 13.9 & 4.59 & $\circ$ & & \\
\hline triglyserides, $\mathrm{mmol} / \mathrm{l}$ & 0.22 & 0.29 & 0.32 & 0.21 & 0.087 & & & $\circ$ \\
\hline glucagon, pg/ml & 100.0 & 71.2 & 91.3 & 77.9 & 21.6 & & $\circ$ & \\
\hline acetoacetate, $\mathrm{mmol} / \mathrm{l}$ & 0.39 & 0.05 & 0.00 & 0.00 & 0.25 & $* *$ & & \\
\hline
\end{tabular}

C9G1: n=2, C12G0 and C12G1: n=3, C9G0: $\mathrm{n}=4$

** as in Table 5 


\section{DISCUSSION}

\section{Multiparous cows}

Silage DMI usually decreases with increased concentrate ration and it has been proposed that substitution ratio increases with higher concentrate levels (Ostergaard, 1979). The substitution ratio between C11G0 and C15G0 in period 1 (Table 8 ) was slightly higher than $-0.48 \mathrm{~kg} \mathrm{DM} / \mathrm{kg}$ DM concentrate for early lactation cows reported by Aston et al. (1995) and $-0.61 \mathrm{~kg} \mathrm{DM} / \mathrm{kg}$ DM concentrate for high concentrate levels in mid lactation reported by Huhtanen (1998).

The addition of glucogenic feed to diet altered the response of cows to concentrate. The substitution ratios between $\mathrm{C} 11 \mathrm{Gl}$ and $\mathrm{C} 15 \mathrm{G} 1$ were close to zero. Since glucogenic feed was a mixture of propylene glycol, polyols, molasses and nicotinamide, it is difficult to say, what is the effect of a separate component of this

TABLE 8

Responses to concentrate supplementation

Responses between

$\mathrm{C} 11 \mathrm{G} 0$ and $\mathrm{C} 15 \mathrm{G} 0 \quad \mathrm{C} 11 \mathrm{Gl}$ and $\mathrm{C} 15 \mathrm{Gl}$

Multiparous cows, period 1

substitution ratio, $\mathrm{kg}$ silage

$-0.88$

$\mathrm{DM} / \mathrm{kg}$ concentrate DM

ECM yicld response,

$-0.40$

$+2.01$

$\mathrm{kg}$ /increased $\mathrm{kg}$ concentrate DM

Multiparous cows, period 2

substitution ratio, $\mathrm{kg}$ silage

$-0.45$

$+0.14$

$\mathrm{DM} / \mathrm{kg}$ concentrate DM

ECM yield response.

$+0.56$

$+0.80$

$\mathrm{kg} /$ increased $\mathrm{kg}$ concentrate DM

C9G0 and $\mathrm{C} 12 \mathrm{G} 0$

$\mathrm{C} 9 \mathrm{Gl}$ and $\mathrm{C} 12 \mathrm{Gl}$

Primiparous cows, period 1

substitution ratio, $\mathrm{kg}$ silagc

$+0.05$

$+0.25$

$\mathrm{DM} / \mathrm{kg}$ concentrate $\mathrm{DM}$

ECM yicld response,

$+0.43$

$+0.42$

$\mathrm{kg} /$ increased $\mathrm{kg}$ concentrate DM

Primiparous cows, period 2

substitution ratio, $\mathrm{kg}$ silage

$+0.09$

$+0.60$

$\mathrm{DM} / \mathrm{kg}$ concentrate DM

ECM yield response,

$+0.11$

$+0.20$

$\mathrm{kg} /$ increased $\mathrm{kg}$ concentrate DM 
mixture on feed intake. In earlier experiments propylene glycol (up to about $500 \mathrm{~g} / \mathrm{d}$ ) ( Fisher et al., 1973; Jans and Münger, 1992) or niacin (12 g/d) (c.g. Dufva et al., 1983) had no significant effect on feed intake. In our experiment, the intakes of propylene glycol and nicotinamide were $244 \mathrm{ml} / \mathrm{d}$ (about $300 \mathrm{~g} / \mathrm{d}$ ) and $6 \mathrm{ml} / \mathrm{d}(7.3 \mathrm{~g} / \mathrm{d})$.

The milk yield response between $\mathrm{C} 11 \mathrm{G} 0$ and $\mathrm{C} 15 \mathrm{G} 0$ was negative during period 1 (Table 8). It must be noted, however, that the substitution ratio between these groups was especially high during this period and thus the increase of ME intake was small. The response during period 2 is in line with Huhtanen (1998), who reviewed some Finnish experiments conducted after peak yield with average concentrate levels $6.72 \mathrm{~kg} \mathrm{DM} / \mathrm{d}$ and $11.57 \mathrm{~kg} \mathrm{DM} / \mathrm{d}$ and with average response of $0.43 \mathrm{~kg} \mathrm{ECM}$ per increased $\mathrm{kg}$ concentrate DM, and it is much higher than the marginal yield $0.05 \mathrm{~kg} / \mathrm{d}$ reported by Østergaard (1979) with a concentrate ration of $11.7 \mathrm{~kg} \mathrm{DM} / \mathrm{d}$ in carly lactation cows. However, Aston et al. (1995) reported much higher milk yield responses with an average response of $1.02 \mathrm{~kg} \mathrm{ECM} \mathrm{per}$ increased $\mathrm{kg}$ concentrate DM with concentrate rations 6.9 and $12 \mathrm{~kg} \mathrm{DM} / \mathrm{d}$.

The milk yield response to concentrate feeding was higher with glucogenic feed than without it. High response to concentrate was due to low milk yield of C11G1 whereas C11G0 had as high milk yield as both high concentrate groups. Using previous lactation $305 \mathrm{~d}$ yicld as a covariate revealed that this was not due to superior milk yield potential of cows in the C11G0. Contrasting some earlier reports of increased milk production with propylene glycol (Emery et al., 1964; Fisher et al., 1973) or niacin (e.g. Dufva et al., 1983), the glucogenic feed with both these ingredients could not increase milk production and in fact $\mathrm{C} 1 \mathrm{lG} 1$ had the lowest milk production. In line with this Jans and Münger (1992) (max $150 \mathrm{~g} / \mathrm{d}$ propylene glycol) or Burhans and Bell (1998) did not observe an increase in milk production of early lactation cows with propylene glycol.

Milk urea concentrations remained under $20 \mathrm{mg} / 100 \mathrm{ml}$ during the whole experiment. Though milk urea concentrations are lower at the early stage of lactation than in other stages (Gustafsson, 1993), the low milk urea concentrations were partly due to the low CP content of silage, which was not totally compensated by inclusion of RSM to the diets. It seems that protein supply, especially the supply of rumen degradable protein, was the limiting factor in C11Gl because $1 \mathrm{~kg}$ concentrate, which had close to zero PBV content, was replaced by low PBV glucogenic feed. PBV values of this group were low and PBV was near $-20 \mathrm{~g} / \mathrm{kg}$ DM during period 2. Therefore rumen microbial protein synthesis and true AAT supply may have been diminished (Table 9). Low rumen degradable protein supply seemed not to limit silage intake capacity of CIIG1 during period 2, but the extra energy partitioned towards liveweight gain.

Milk protein content and protein yield have increased in several experiments (Gordon et al., 1984; Thomas et al., 1986; Aston et al., 1995) with increasing 
concentrate as a result of higher energy intake. However, the biggest increases in protein content have been achieved between low levels of concentrate ( 3 to $6 \mathrm{~kg}$ $\mathrm{DM} / \mathrm{d}$ ) (Aston et al., 1995). This may explain the fact that concentrate level had no effect on milk protein content in our experiment with high concentrate levels.

Encrgy utilisation was lower in $\mathrm{C} 15$ groups than in $\mathrm{C} 11$ groups, which reflects the diminishing returns with increased concentrate. In contrast to carlier reports (Østergaard, 1979; Gordon, 1984; Aston et al.. 1995), liveweight loss secmed to be greater at higher concentrate level, although NEFA concentration of plasma gives no support to LWC data. It is possible that the greater liveweight loss was due to greater fat deposits at the beginning of lactation. Condition score of C15 group was higher than that of $\mathrm{C} 11$ group three weeks before calving.

Glucagon may stimulate glycogenolysis and gluconcogenesis in liver and it may increase the usage of amino acids to gluconeogenesis (Brockman and Laarveld, 1986). There was a strong positive correlation between ECM and plasma glucagon concentration (means of first six lactation weeks) ( $\mathrm{r}=0.67, \mathrm{P}=0.005)$. which reflects the dependence of multiparous cows in carly lactation on gluconeogenesis. Plasma glucagon concentrations were higher at the higher level of concentrate in our experiment. Glucose concentrations were not significantly affected but on average they were higher with higher concentrate level. The results reported by Aiello et al. (1984) give some support to increased gluconeogenesis at the higher level of concentrate. They observed higher rates of gluconeogenesis with liver samples taken from cows having high concentrate dict than in samples taken from cows having low concentrate diet. Furthermore, there is some evidence that the capacity of liver to convert propionate to glucose is higher with increased energy intakes (Overton, 1998).

Propylene glycol and niacin are both components capable of decreasing blood ketone concentrations (Sauer et al.. 1973; Dufva et al., 1983; Jans and Münger, 1992) and propylene glycol has been shown to increase blood glucose concentrations (Emery et al., 1964). This trend is seen in our experiment. However, it must be noted that using the milk acetone threshold $(>0.40 \mathrm{mmol} / \mathrm{l})$ presented by Andersson (1988) multiparous cows had only threc hyperketonaemic milk samples: one each in groups $\mathrm{C} 11 \mathrm{G} 0, \mathrm{C} 15 \mathrm{G} 0$ and $\mathrm{C} 15 \mathrm{Gl}$.

\section{Primiparous cows}

As in older cows, the inclusion of glucogenic feed altered the response of cows to the higher level of concentratc. The increasc of silage DM intake with increased concentrate was higher between $\mathrm{C} 9 \mathrm{Gl}$ and $\mathrm{C} 12 \mathrm{Gl}$ than between $\mathrm{C} 9 \mathrm{G} 0$ and $\mathrm{C} 12 \mathrm{G} 0$ during both periods (Table 8). Nevertheless, all substitution ratios were positive. Positive substitution ratio (i. e. silage intake increases with concentrate intake) is an exception (Thomas, 1987). In contrast to our results Østergaard (1979) 
observed that substitution ratio was more negative with primiparous cows than with multiparous cows.

Differing from multiparous cows C9G0 had the lowest milk yicld although without significant differences. C9G1 had as high a milk yield as the two higher concentratc groups. The differences between the groups in ECM were smaller. The average response of ECM yicld to increased concentrate (Table 8) was low but not as low as in Østergaard's (1979) experiment. where the marginal yield was negative when heifers were fed $11.7 \mathrm{~kg} \mathrm{DM} / \mathrm{d}$ concentrate. At lower concentrate levels heifers have had better responses to increased concentrate (Phipps et al., 1987; Coulon et al., 1994). In experiment reported by Phipps et al. (1987) the response was $0.62 \mathrm{~kg}$ milk per increased $\mathrm{kg}$ concentrate DM.

Though CP and AAT intakes were higher in $\mathrm{C} 12$ than in $\mathrm{C} 9$. milk yield response remained low. Protein or amino acid supply (Table 9) was probably not the primary limiting factor for milk production. In this respect there seems to be a difference between primiparous and multiparous cows. The lower response of heifers to the higher level of concentrate compared to older cows probably reflects a difference in milk yicld potential. Primiparous cows have a rather high priority for tissue gain and they may not therefore mobilize adipose tissues as efficiently as older cows (Strickland and Brostcr, 1981).

TABLE 9

PBV corrected $\triangle \wedge \mathrm{T}$ intakes

\begin{tabular}{|c|c|c|c|c|}
\hline & $\mathrm{C} 11 \mathrm{G} 0$ & $\mathrm{C} 11 \mathrm{Gl}$ & C.15G0 & $\mathrm{C} 15 \mathrm{Gl}$ \\
\hline \multicolumn{5}{|c|}{ Multiparous cows, period 1} \\
\hline $\mathrm{PBV}, \mathrm{g} / \mathrm{kg} \mathrm{DM}$ & -13.0 & -16.4 & -12.4 & -13.7 \\
\hline true $\Lambda \Lambda \mathrm{T}$ intake, $g / \mathrm{d}^{\prime \prime}$ & 1729 & 1582 & 1816 & 1857 \\
\hline \multicolumn{5}{|c|}{ Multiparous cows, period 2} \\
\hline $\mathrm{PBV}, \mathrm{g} / \mathrm{kg} \mathrm{DM}$ & -14.0 & -19.6 & -15.0 & -19.0 \\
\hline \multirow[t]{2}{*}{ true $A \wedge T$ intake, $g / d$} & 1777 & 1694 & 2039 & 2093 \\
\hline & $\mathrm{C} 9 \mathrm{GO}$ & $\mathrm{C} 9 \mathrm{G} 1$ & $\mathrm{C} 12 \mathrm{G} 0$ & C $12 \mathrm{G} 1$ \\
\hline \multicolumn{5}{|c|}{ Primiparous cows, period 1} \\
\hline $\mathrm{PBV}, \mathrm{g} / \mathrm{kg} \mathrm{DM}$ & -14.8 & -18.5 & -16.6 & -20.3 \\
\hline true $\triangle A T$ intake, $g / d$ & 1307 & 1189 & 1529 & 1447 \\
\hline \multicolumn{5}{|c|}{ Primiparous cows, period 2} \\
\hline PBV, g/kg DM & -11.9 & -14.8 & -10.3 & -14.6 \\
\hline true AAT intake, g/d & 1428 & 1302 & 1754 & 1709 \\
\hline
\end{tabular}

I true $A \wedge T$ intake $(\mathrm{g} / \mathrm{d})=$ AAT intake $(\mathrm{g} / \mathrm{d})+0.595 \times$ PBV $(\mathrm{g})$ (Ttori et al., 1996), assuming the proportion of amino acids in microbial protein $=0.70$ and the digestibility of microbial protein $=0.85$ 
There were positive correlations between ECM and NEFA and ECM and BHB in multiparous cows $(\mathrm{r}=0.42, \mathrm{P}=0.11 ; \mathrm{r}=0.62, \mathrm{P}=0.01)$ but not in primiparous cows $(\mathrm{r}=0.09, \mathrm{P}=0.75 ; \mathrm{r}=0.01, \mathrm{P}=0.97)$. This suggests smaller role of tissue deposits for milk production in primiparous cows.

It seems that the heifers in our experiment were able to reach their maximal yield with $9 \mathrm{~kg} / \mathrm{d}$ concentrate. This seems to indicate that some physiological factor, perhaps the capacity of secretory cells in the mammary gland may have limited milk production. Furthermore, there was no positive correlation between glucagon and milk yield in primiparous cows.

Plasma glucose concentrations in C9G0 at weeks 4 and 6 were lower than in other groups suggesting that glucose supply may have limited milk yield in this group. Morcover, using the threshold of milk acctone $(>0.40 \mathrm{mmol} / \mathrm{l})$ presented by Andersson (1988), the only ketotic animal in the experiment was in this group.

Plasma glucose seemed to be higher and ketone concentrations seemed to be lower at the higher level of concentrate. With glucogenic feed this trend can be seen too.

The extra energy from concentrate was partitioned towards liveweight gain during period 2. This is in line with earlier experiments (Østergaard, 1979; Phipps et al., 1987; Coulon et al., 1994). In line with liveweight data plasma insulin was higher at the higher level of concentrate 6 wecks after calving, C12G1 having the highest concentrations and C9G1 having the lowest concentrations. As in older cows, the energy utilisation for milk production was lower at the higher level of concentrate.

\section{CONCLUSIONS}

Due to the experimental design even fairly large differences (e.g. milk yield difference of $3 \mathrm{~kg} / \mathrm{d}$ ) proved to be statistically not significant. This design was dictated by our desire to study the whole period of carly lactation, including the first week after parturition, which is often neglected in experiments resembling that of ours.

In both parity groups, maximal milk yicld during the experiment could be achieved even with one of the treatments with lower concentrate level. However. there was a difference in response to glucogenic feed between parities. In older cows, glucogenic feed had a negative effect on milk production at the lower concentrate level, suggesting that with low $\mathrm{CP}$ grass silage rumen degradable protein was the factor limiting milk yield instead of supply of glucogenic substrate. Due to lower production potential of primiparous cows, their amino acid need for milk production could be satisfied even with lower level of concentrate with glucogenic feed. 
Plasma glucose concentrations of multiparous cows were higher and the concentrations of ketones were lower with glucogenic feed. In heifers, after peak yield, the plasma insulin concentrations were higher with the higher concentrate level. This was accompanied by partitioning extra nutrients towards tissue gain.

\section{ACKNOWLEDGEMENTS}

The authors wish to thank M. Sc. Sakari Alasuutari, Mr. Jorma Tossavainen and the staff of Suitia research farm for conducting the trial and DVM Juha Kallio for the interpretation of progesterone analyses.

\section{REFERENCES}

Aiello R.J., Kenna T.M., Herbein J.H., 1984. Hepatic gluconeogenic and ketogenic interrelationships in the lactating cow. J. Dairy Sci. 67, 1707-1715

Andersson L., 1988. Subclinical ketosis in dairy cows. Vet. Clin. North Amer. Food Anim. Prac. 4, $233-251$

Aston K., Sutton J.D., Fisher W.J., 1995. Milk production from grass silage diets: strategies for concentrate allocation. Anim. Sci. 61, 465-480

Brockman R.P., Laarveld B., 1986. Hormonal regulation of metabolism in ruminants; a review. Livest. Prod. Sci. 14, 313-334

Burhans W.S., Bell A.W., 1998. Feeding the transition cow. In: Proceedings of 1998 Cornell Nutrition Conference for Feed Manufacturers, pp. 247-258

Coulon J.B., D'Hour P., Garel J.P., Petit M., 1994. Level and pattern of winter concentrate allocation in dairy cows: results in first lactation cows. Anim. Prod. 59, 11-20

Dufva G.S., Bartley E.E., Dayton A.D., Riddell D.O., 1983. Effect of niacin supplementation on milk production and ketosis of dairy cattle. J. Dairy Sci. 66, 2329-2336

Edmonson A.J., Lean I.J., Weaver L.D., Farver T., Webster G., 1989. A body condition chart of Holstein dairy cows. J. Dairy Sci. 72, 68-78

Emery R.S., Burg N., Brown L.D., Blank S.N., 1964. Detection, occurrence and prophylactic treatment of borderline ketosis with propylene glycol feeding. J. Dairy Sci. 47, 1074-1079

Fisher L.J., Erfle J.D., Lodge G.A., Sauer F.D., 1973. Effects of propylene glycol or glycerol supplementation of the diet of dairy cows on the feed intake, milk yield and composition, and incidence of ketosis. Can. J. Anim. Sci. 53, 289-296

Gordon F.J., 1984. The effect of level of concentrate supplementation given with grass silage during the winter on the total lactation performance of autumn-calving cows. J. Agr. Sci. 102, 163-179

Gustafsson A.H., 1993. Acetone and urea concentrations in milk as indicators of the nutritional status and the composition of the diet of dairy cows. Academic Dissertation. Report 222, Swedish University of Agricultural Science, Department of Animal Nutrition and Management

Huhtanen P., 1998. Supply of nutrients and productive responses in dairy cows given diets based on restrictively fermented silage. Agr. Food Sci. Finland 7, 219-250

Jans F., Münger A., 1992. Effet du propylèneglycol comme agent de prevention de l'acétonémie durant la phase de démarrage chez la vache laitière. Revue Suisse Agr. 24, 237-242 
Kokkonen T., Tuori M., Leivonen V., Syrjälä-Qvist L., 2000. Effect of silage dry matter content and rapeseed meal supplementation on dairy cows. 1. Milk production and feed utilisation. Anim. Feed Sci. Tech. 84. 213-228

Laitinen J., 1982. Evaluation of defatted and whole milk progesterone radioimmunoassays (in Finnish). Suomen cläinlääkärilehti $88,501-505$

MAFF, 1975. Energy Allowance and Feeding System for Ruminants. Tech. Bull. 33. Her Majesty's Stationery Office, London

McCutcheon S.N., Bauman, D.E., 1986. Effect of chronic growth hormone treatment on responses to epinephrine and thyrotropin releasing hormone in lactating cow. J. Dairy Sci. 69, 44-51

Østergaard V.. 1979. Stategies for concentrate feeding to attain optimum feeding level in high yiclding dairy cows. Beretning fra Statens Husdyrbrugsforsøg, No. 482

Overton $T_{.}$1998. Substrate utilisation for hepatic gluconeogenesis in the transition dairy cow. In: Proceedings of 1998 Cornell Nutrition Conference for Feed Manufacturers, pp. 237-246

Phipps R.H., Weller R.F.. Bines J.A., 1987. The influence of forage quality and concentrate level on dry matter intake and milk production of British Friesian heifers. Grass Forage Sci. 42, 49-58

Rajamäki S., Rauramaa A., 1985. The determination of acetoacetate and 3-hydroxybutyrate in milk. Finn. J. Dairy Sci. 43, 9

- SAS, 1989. SAS/STAT Users Guide, Version 6. Vol. 2. $4^{\text {th }}$ Edition. SAS Institute Inc., Cary. NC (USA)

Sauer F.D., Erfie J.D., Fisher L.J., 1973. Propylene glycol and glycerol as a feed additive for lactating dairy cows: an evaluation of blood metabolite parameters. Can. J. Anim. Sci. 53, 265-271

Sjaunja L.O.. Baerve L.. Junkkarinen L., Pedersen J., Setälä J., 1990. A Nordic proposal for an energy corrected milk (ECM) formula. In: 27 th Session International Committee of Recording and Productivity of Milk Animal, Paris (France), pp. 156-157

Strickland M.J.. Broster, W.H., 1981. The effect of different levels of nutrition at two stages of the lactation on milk production and live-weight change in Friesian cows and heifers. J. Agr. Sci. 96. 677-690

Thomas $\mathrm{C} ., 1987$. Factors affecting substitution rates in dairy cows on silage based rations. In: W. Haresign. D.J.A. Cole (Editors). Recent Advances in Animal Nutrition. Butterworths, London, pp. 205-218

Thomas C.. Aston K., Daley S.R., Bass J., 1986. Milk production from silage. 4. The effect of the composition of the supplement. Anim. Prod. 42, 315-325

Trinder P., 1969. Determination of glucose in blood using glucoseoxidase with an altemative oxygen acceptor. Ann. Clin. Biochem. 6, 24-27

Tuori M., Kaustell K., Valaja J., Aimonen E., Saarisalo E., Huhtanen P., 1996. Feed tables and feeding recommendations. 2nd Edition. Helsinki (Finland)

Työppönen J., Kauppinen K., 1980. The stability and automatic determination of ketone bodies in blood samples taken in field conditions. Acta Vet. Scand. 21, 55-61

Wahlefeld A.W. 1974. Triglycerides. Determination after enzymatic hydrolysis. In: H.U. Bergmeyer, (Editor). Methods of Enzymatic Analysis. Vol. 4. 2nd English Edition. Academic Press. New York 


\section{STRESZCZENIE}

Wplyw ilości paszy treściwej oraz paszy glukogennej podawanych we wczesnym okresie laktacji na pobranie paszy, produkcję mleka oraz przemianę energii u krów mlecznych i jałówek

W doświadczeniu, o układzie czynnikowym $2 \times 2$, przeprowadzonym na krowach fryzach, 16 wieloródkach i 16 pierwiastkach, porównano dwa poziomy paszy treściwej oraz wpływ dodatku paszy glukogennej $(0$ l; G0 lub l l/d Gl) na wyniki produkcyjnc. llość paszy treściwej dla wieloródek wynosiła $11 \mathrm{~kg} / \mathrm{d}(\mathrm{C} 11)$ lub $15 \mathrm{~kg} / \mathrm{d}$ (C15), dla pierwiastek $9 \mathrm{~kg} / \mathrm{d}$ (C9) lub $12 \mathrm{~kg} / \mathrm{d}$ (C12). Pasza glukogenna składata się z glikolu propylenowego, polialkoholi, melasy i niacyny. Ponadto wszystkie zwierzęta otrzymywały do woli podwiędniętą kiszonkę. Doświadczenic trwało 12 tygodni, rozpoczynając od wyciclenia krów.

Przy podawaniu paszy glukogennej krowom wieloródkon stwicrdzono niewielki wpływ dawki na pobranie s.m. kiszonki, podezas gdy bez tego dodatku krowy zjadały więcej s.m. paszy treściwej zamiast s.m. kiszonki. Pasza glukogenna miała też niewiclki wpływ na produkcję mleka przy wiçkszych dawkach paszy treściwej, natomiast ujemny - przy mniejszej dawce. W wyniku tej interakcji rcakcja wydajności mleka na większą dawkę paszy treściwej byla większa przy dodatku paszy glukogennej. Przyczyną tych interakcji była prawdopodobnie mała ilość białka rozkładancgo w żwaczu przy skarmianiu dawki C1! Gl.

U pierwiastek reakcja, wyrażona ilością produkowancgo mleka na większą dawkę paszy treściwej. była mała. Po szczycie wydajności mleka stężenie insuliny w plazmie krwi, podobnic jak i przyrosty dzienne były większe przy większej dawce paszy treściwej.

W podsumowaniu stwicrdzono, że stopień reakcji wicloródck na wysokic dawki paszy treściwej zależy od podaży ilości rozkładanego w żwaczu białka. podczas gdy u pierwiastek jest ograniczona zdolnością produkcji mleka i zapotrzebowaniem na wzrost. Dodatek paszy glukogennej zwiększa poziom glukozy, a obniża stężenie ciał ketonowych w plazmie krwi. 